\title{
Phylogenetic relationships of the European newts (genus Triturus) tested with mitochondrial DNA sequence data
}

\author{
I. Zajc ${ }^{1} \&$ J. W. Arntzen ${ }^{2}$ \\ Department of Zoology, Leicester University, Leicester LE1 7RH, United Kingdom \\ Present addresses: 'National Institute of Biology, Vecna pot 111, P.O. Box 141, 1001 Ljubljana, Slovenia \\ ${ }^{2}$ Department of Zoology and Anthropology, Faculty of Sciences, University of Porto, CECA/ICETA/UP, \\ Campus Agrário de Vairão, 4480 Vila do Conde, Portugal
}

Keywords: 12S rRNA, ATPase, mitochondrial DNA, newt, phylogeny, Triturus

\begin{abstract}
European newts (genus Triturus) are widely studied, but their phylogeny is not yet unambiguously resolved. Fragments of mitochondrial DNA experiencing different rates of evolution (the ATPase and 12S rRNA genes) were sequenced in order to test a phylogenetic hypothesis derived from biochemical and behavioral data. Well-supported branches of the existing phylogeny gained support in our study. The monophyletic origin of the hypothesized $T \times$ boscai $-T$. italicus clade remained ambiguous, whereas strong support was gained for the sister-taxon relationship of $T$, vulgaris and $T_{x}$ montandoni. The position of $T$. vittatus as a sister taxon to the $T$. marmoratus species group was also supported. The phylogenetic position of $T$, alpestris could not be clarified. With an in-group taxon sampling denser than in previous molecular phylogenetic studies and under the a priori selection of species from the genera Cynops, Neurergus and Paramesotriton as out-groups, the monophyly of Triturus was strongly supported. It cannot be excluded, however, that the presumed out-group actually belongs to the in-group, rendering Triturus paraphyletic as was concluded from recently published $12 \mathrm{~S}$ and 16S rRNA sequence data.
\end{abstract}

\section{Contents}

Abstract

Introduction

Materials and methods

Results

Discussion

Acknowledgements

References

\section{Introduction}

Phylogenies are a cornerstone to the study of evolution: without them we are unable to reconstruct and understand pattern and process of evolutionary change. To obtain a robust phylogenetic hypothesis requires the gathering of multiple independent and complementary data sets, because usually no single data set is sufficiently powerful to simultaneously resolve older and more recent cladogenetic events.

The genus Triturus (European newts) has been the subject of extensive phylogenetic analysis with various data sets [osteological (Bolkay, 1928; Rafinski and Pecio, 1989), immunological (Busack et al., 1988), morphological (Giacoma and Balletto, 1988), biochemical (Rafinski and Arntzen, 1987), behavioral (Arntzen and Sparreboom, 1989), cytogenetic (Macgregor et al., 1990)], and is considered by some to be the phylogenetically best studied genus in the world (Halliday and Arano, 1991). Despite these concerted efforts, its phylogeny is not fully resolved and several competing hypotheses are available, none of them with unambiguous overall support. We take the study by Arntzen and Sparreboom (1989) as the basis of our work because the phylogeny they present is based on two independent and complementary data sets. Moreover, their hypothesis resolves the earlier as well as the later events in the Triturus radiation and is robust under the jack-knife test. 


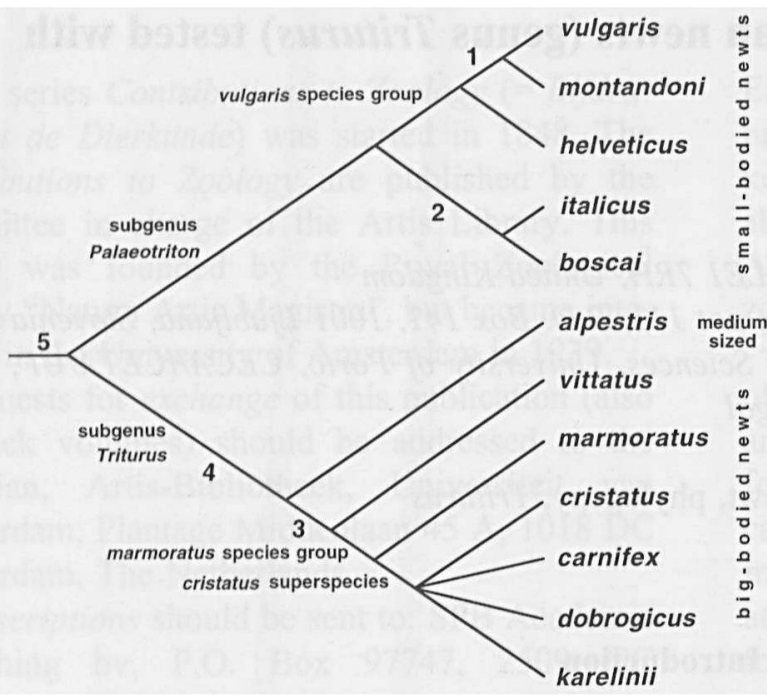

Fig. 1. Phylogeny for the genus Triturus based on available biochemical and behavioral characters. Numbers refer to specific questions as addressed in the text. Out-group taxa are taken from the genera Cynops, Neurergus, and Paramesotriton.

Nine or twelve Triturus species are currently recognized, depending on the criteria used for species recognition. Here we refer to twelve species, with four of them (Triturus cristatus, T. carnifex, T. karelinii and $T$. dobrogicus) grouped together in the T. cristatus superspecies (Wallis and Arntzen, 1989). The 'big' - and 'medium-sized' newts ( $T$. cristatus, T. marmoratus, $T$. vittatus and T. alpestris) are organized in the subgenus Triturus and the 'small-bodied' newts ( $T$. boscai, $T$. helveticus, $T$. italicus, T. montandoni and T. vulgaris) are placed in the subgenus Palaeotriton. The detailed con $\nmid$ figuration is given in Fig. 1, alongside with the nomenclature that we adopt. For the following sections of the phylogenetic tree the support is limited or contradictory: 1) the sister-taxon status of $T$. vulgaris and $T$. montandoni is supported by allozyme data only; 2) the monophyly of $T$. boscai and $T$. italicus hinges on the interpretation given to behavioral characters; 3 ) the status of $T$. vittatus as the sister taxon of the $T$. marmoratus species group may be called into question on the basis of its overall similarity to some small-bodied species, $T$. vulgaris in particular; 4) the support for the position of $T$. alpestris in the subgenus Triturus is relatively weak due to the non-independence of some behavioral synapomorphies; 5) the monophyly of Triturus, traditionally taken for granted, has been put in doubt by molecular data (Titus and Larson, 1995).

We tested the phylogenetic hypothesis of Triturus with a newly generated set of independent data. Two fragments of the mitochondrial (mt)DNA molecule experiencing different evolutionary rates were studied. The slowly evolving 12S rRNA gene (Kocher et al., 1989; Hickson et al., 1996) was partially sequenced to test some of the supposedly earlier events of the Triturus radiation, while the fast evolving ATPase gene (Kumar, 1996) was partially sequenced to test the branching order among some supposedly closely related species in the T. vulgaris species group. Sequence data for cytochrome $b$ produced ambiguous phylogenetic results, perhaps due to the comparison of nonhomologous sequences (Caccone et al., 1997; cf. Zhang \& Hewitt, 1996) and were discarded.

\section{Materials and methods}

Two specimens were selected from each of nine Triturus species, if possible from different localities. As out-groups to the genus, representatives of the salamandrid genera Cynops, Neurergus and Paramesotriton were selected, following Arntzen and Sparreboom (1989). Triturus boscai was taken as out-group to the T. vulgaris species group. Specimens for which DNA was extracted were sampled as follows: $T$. alpestris from Mayenne, France and Pola de Sierra, Spain (subspecies cyreni); T. boscai from Toledo, Spain; T. cristatus from Limanowa, Poland and Sinaia, Romania; T. helveticus from Ambleteuse, France and Canterbury, U. K.; T. italicus from Conversano, Italy; $T$. marmoratus from El Berrueco near Madrid, Spain and Rochechouart, France; $T$. montandoni from Ustrzyki, Poland; $T$. vittatus from an unknown locality in Israel and from Adapazari, Turkey (subspecies ophryticus) and T. vulgaris from Ambleteuse, France. Outgroup taxa Cynops ensicauda, Neurergus strauchii and Paramesotriton sp. were obtained from the pet trade with no reliable locality information.

Total DNA was extracted from approximately $50 \mathrm{mg}$ of frozen or ethanol-preserved tissue (liver, 
heart or tail muscle) following standard protocols (Sambrook et al., 1989). For PCR-amplification of the 12S rRNA region the forward (L2475) and reverse (H2897) 'universal' primers were used (Kocher et al., 1989). The forward and reverse ATPase primers L9858 (5'-CTCCTCCTTAATGATATGCCACA-3') and H10307 (3'-TTCACCCCAACTACCCAACTATC-5') were designed in our laboratory by G. Rowe. Double stranded DNA amplifications were performed using standard PCR protocols (Erlich, 1989) with negative controls to test for contamination. The 12S rRNA fragment was amplified under stringent conditions (annealing temperature $65^{\circ} \mathrm{C}$, extension time 25 seconds, up to 28 cycles), while the ATPase primers required milder conditions (annealing temperature $45-50^{\circ} \mathrm{C}$, extension time of 60 seconds, minimum of 30 cycles). The amplified products were separated on $1.5 \%$ low melting point agarose gels, cut out of the gel and purified by successive phenol, phenol-chloroform and chloroform-isoamylalcohol extractions, followed by ethanol precipitation. Both strands were directly sequenced using the dideoxy chain-termination method (Sanger et al., 1977). Multiple sequences were aligned with Clustal-V software (Higgins and Sharp, 1988) with manual adjustments. The ATPase gene sequences were translated into amino-acid sequences and the reading frame was determined by alignment to sequences of the teleost fish Cottus kessleri (Grachev et al., 1992) and human sequences (Arnason et al., 1996) obtained from GenBank. When the sequences for individuals of the same species were not identical, the consensus sequence was used in the phylogenetic analysis with variable sites taken as polymorphisms. The percent sequence divergence was calculated following Mindell and Honeycutt (1990). We aligned our sequences to the published salamandrid 12S sequences (Caccone et al., 1994; Hay et al., 1995), that include the in-group taxa $T_{x}$ vulgaris and $T_{x}$ carnifex and outgroup taxa from the genera Euproctus, Notophthalmus, Pleurodeles and Salamandra. Furthermore, the sequences were aligned to those published by Titus \& Larson (1995), that include T. alpestris, I. karelinii and representatives of the salamandrid genera Chioglossa, Cynops, Euproctus, Neurergus, Mertensiella, Notophthalmus, Pachytriton, Para- mesotriton, Pleurodeles, Salamandra, Salamandrina, Taricha and Tylototriton.

Phylogenetic reconstruction was performed under the principle of parsimony, using the PAUP 3.1.1 software (Swofford, 1991) with the 'branch-andbound' search option (the 'heuristic search' method was used in the bootstrap runs). Two a priori selected strategies were followed. First, a uniform transition - transversion ratio and uniform positional ratio was used, i.e., 'no weighting'. Second, different weights were assigned to transitions versus transversions inversely proportional to the frequency of different substitutions among in-group taxa as traced from the tree obtained with the unweighted approach, with 24 as the base number. Gap derived character-state changes were given the same weight as transversions, also on an a priori basis. Positional weighting factors for the first, second, and the third codon positions were determined for the protein coding DNA fragment (ATPase) in a similar way. Bootstrap scores (Felsenstein, 1985; Hillis and Bull, 1993) were determined over 5000 replications to obtain an impression of the strength of support from the data for the phylogenetic tree showing maximum parsimony. Templeton's (1983) non-parametric test was used to test one-sided for the significance of differences between documented phylogenies and the newly derived alternatives.

\section{Results}

Aligned sequences represent $212 \mathrm{bp}$ of the ATPase gene and covers the short stretch of overlap between regions coding for ATPase 6 and ATPase 8 (Fig. 2). The first nucleotide corresponds to position 256 in the Cottus kessleri ATPase sequence (Grachev et al., 1992). Aligned sequences represent $301 \mathrm{bp}$ of 12S rRNA gene. The first nucleotide corresponds to position 233 in the Mertensiella luschani 12S rRNA sequence (Titus and Larson, 1995). No insertions or deletions ('indels') had to be inferred to align the ATPase sequences. Alignment of the 12S sequences required two indels at one position among the Triturus species and two indels covering four positions in the out-group taxa. More indels on nine positions were required when 


\begin{abstract}
01 CAGATACTA CGAGCAACAG CTTAAAACTC AAAGGACTTG GCGGTGCTCT ACACCCCCCT AGAGGAGCCT GTTCTATAAT CGATAATCCA CGATAAACCT 02 CGGAACACTA CGAACAACAG CCTAAAACTC AAAGGACTTG GCGGTGCTCT ACACCCACCT AGAGGAGCCT GTTCTATAAT CGATAATCCA CGATAAACCT 03 CAGAACACTA CGAGCAACAG CTTAAAACTC AAAGGACTTG GCGGTGCTCC ACACCCCCCT AGAGGAGCCT GTTCTATAAT CGATATTCCT CGATAAACCT 04 CAGAAYACTA CGAGCAACAG CTTAAAACTC AAAGGACTTG GCGGTGCTCT ACACCCCCCT AGAGGAGCCT GTTCTATAAT CGAYAMCCCC CGATAAACCT 04 CAGAAYATA CGAGCAACAG CTTAAAACTC AAAGGACTTG GCGGTGCTCT ACACCCCCCT AGAGGAGTA CGAGCAACAG CTTAAAACTC AAAGGACTTG GCGGTCTCT ACACCCCCCT AGAGGAGCCT GTTCTATAAT CGATAACCCC CGATAAACCT 06 CAGAGTACTA CGAGCAACAG CTTAAAACTC AAAGGACTTG GCGGTGCTCT ACACCCACCT AGAGGAGCCT GTTCTGTAAT CGATAATCCA CGATAAACCT 07 CAGAGTACTA CGAGCAACAG CTTAAAACTC AAAGGACTTG GCGGTGCTCT ATACCCCCCT AGAGGAGCCT GTTCTATAAT CGATAATCCC CGATAAACCT 08 CAGAATACTA CGAGCAACAG CTTAAAACTC AAAGGACTTG GCGGTGCTCT ACACCCCCCT AGAGGAGCCT GTTCTATAAT CGATAATCCC CGATAAACCT 08 CAGAATACTA CGAGCAACAG CTTAAAACTC AAAGGACTTG GCGGTGCTCT ACACCCCCCT AGAGGAGCCT GITCTATAAT CGATAATCCC CGATAAATATATATA CGATATAT 09 CAGAGTACTA CGAGCAACAG CTTAAAACTC AAAGGACTTG GCGGTGCTCT ACACCCCCCT AGAGGAGCCT GTTCTATAAT CGATAATCCA CGATAAACCT 10 CAGAGTACTA CGAGCAACAG CTTAAAACTC AAAGGACTTG GCGGTGCTCT ACACCCCCCT AGAGGAGCCT GTTCTATAAT CGATAATCCC CGATAAACCT 11 CAGAGTACTA CGAGCAACAG CTTAAAACTC AAAGGACTTG GCGGTGCTCT ACACCCCCCT AGAGGAGCCT GTTCTATAAT CGATAATCCA CGATAAACCT

12 CAGAGTACTA CGAGCAACAG CTTAAAACTC AAAGGACTTG GCGGTGCTCT ATACCCCCCT AGAGGAGCCT GTTCTATAAT CGATAATCCC CGATAAACCT

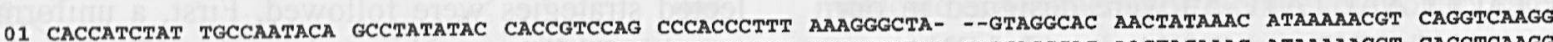
02 CACCATCTGT TGCTAATACA GCCTATATAC CACCGTCCAG CCCACCCTCT AAgGgTATAA TAGTAGGCAC AACTACAAAC ATAAAAACGT CAGGTCAAGG 03 CACCATCTAT TGCCAATACA GCCTATATAC CACCGTCCAG CCCACCCTTC AAAGGACTAA CAGTAGGCAC AACTATAAAC ATAAAAACGT CAGGTCAAGG 04 CACCATCTAT TGCYARTACA GCCTATATAC CACCGTCCAG CCCACCCTTT AAAGGRWAA YAGTAGGCAC AAYTATAGAC ATAAAAACGT CAGGTCAAGG 05 CACCATCTGT TGCCAATACA GCCTATATAC CACCGTCCAG CCCACCCTTT AAGGGTAAAA CAGTAGGCAC AACTATAGAC ATAAAAACGT CAGGTCAAGG 05 CACCATCTGT TGCCAATACA GCCTATATAC CACCGTCCAG CCCACCCTTI AAGGGTAAA CAGTAGGCAC AACTATACAC ATAAGAACGT CAGGTCAAGG 06 CACCACTTGT TGCCAATACA GCCTATATAC CACCGTCCAG CCCACCCTTC AAAGGGTAA AAGTAGGCAA AATTATACAC ATAAGAACGT CAGGTCAAGG 07 CACCATCTAT TGCCAATACA GCCTATATAC CACCGTCCAG CCCACCCTTT AAAGGAAAGA CAGTAGGCAC AACTACACAC ATATAAACGT CAGGTCAAGG 08 CACCATCTAT TGCCAATACA GCCTATATAC CACCGTCCAG CCCACCCTTT AAAGGTAAAA CAGTAGGCAC AACTATACAC ATAAAAACGT CAGGTCAAGG 09 CACCACTTAT TGCCAATACA GCCTATATAC CACCGTCCAG CCCACCCTTC AAGGGACCAA AAGTAGGCAC AATTATACAC ATAAAAACGT CAGGTCAAGG 10 CACCATCTAT TGCCAATACA GCCTATATAC CACCGTCCAG CCCACCCTTT AAAGGAAAG CAGTAGGCAC AACTACACAC ATATAAACGT CAGGTCAAGG 11 CACCATTTAT TGCTAATACA GCCTATATAC CACCGTCCAG CCCACCCTTT AAGGGTCAAA YAGTAGGCAT AATTATAGAC ATARAAACGT CAGGTCAAGG 12 CACCATCTAT TGCCAATACA GCCTATATAC CACCGTCCAG CCCACCCTTT AAAGGAAAGA CAGTAGGCAC AACTACATAC ATATAAACGT CAGGTCAAGG

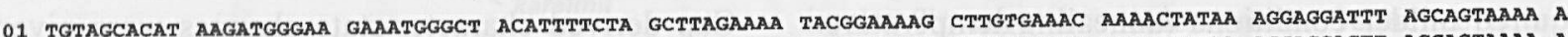
02 TGTAGCAAAT AAGATCGGAA GAAATAGGCT AC-TTTGCTA CCTTGAAAAA TACGGAAAAG CTTGTGAAAC AAAACTATAA AGGAGGACTT AGCAGTAAAA A 02 TG 03 TGTAGCATAT AAGATGGGAA GAAATGGGCT ACATTTTCTA ACTTAGAAAA TACGGAAAAG CTTGTGCAAC AAAACTATAA AGGAGGATTT AGCAGTAAGA A 04 TGTAGCGTAT GAGATGGGA GAAATGGCT ACATTTTCTA ACCTAGAAAA CACGGAAAAG TTTATGAAAC AAAACTATAA AGGAGGATTT AGTAGTAAAA A 05 TGTAGCAGAT AAGATGGGAA GAAATGGGCT ACATTTTCTA ACCTAGAAAA CACGGAGAAg TTTATGAAAC TAAACTATAA AGGAGGATT AGTAGTAAAA A 06 TGTAGCAAAT AAAGCGGGA GAAATGGGCT ACATTTTCTA ATCTAGAAAA CACGGAAAAg TCTGTGAAAT AGAACTACAA AGGAGGATTC AGAAGTAAAA A 07 TGTAGCAAAT AAGATGGGA GAAATGGGCT ACATTTTCTA A-CTAGAAAG CACGGAAAAG TTTATGAAAC TAAGCTATGA AGGAGAATTT AGCAGTAAAA A 08 TGTAGCAGAT AAGATGGGA GAAATGGGCT ACATTTTCTA ACCTAGAAAA CACGGAAGAg TCTATGAAAC TAAACTATGA AGGAGGATTT AGCAGTAAAA A 18 TAGCAGAT AAGATGGGA GAAATGGCT ACATTTTCTA ACCTAGAAAA TACGGAAAAG TCTATGAAAT AAAACTACGA AGGAGGATTT AGCNGTAAAA A

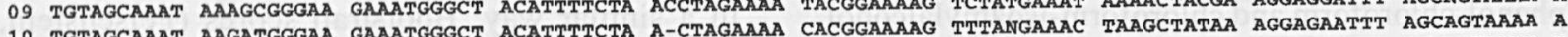
10 TGTAGCAAAT AAGATGGGAA GAAATGGGCT ACATTTTCTA A-CTAGAAAA CACGGAAAAG TTTANGAAAC TAAGCTATAA AGGAGAATT AGCAGTAAAA A 11 TGTAGCAAAT AAGATGGGA GAAATGGGCT ACATTTTCTA AYCTAGAAAA CACGGAAAAG TCYATGAAAC AGRACTATAA AGGAGGATT AGAAGTAAAA A 12 TGTAGCAAAT AAGATGGGA GAAATGGGT ACATTTTCTA ACCTAGAAAA CACGgAAAA TTTATGAAAC TAAGCTATAA AGgAGAATT AGCAGTAAAA A
\end{abstract}

***

05 ACACAACCCT GAAACTGACC ATGAATTTAG GCTTTTTTGA CCAATTTATA AGCCCCACCG TACTAGGCAT TCCTTTAATC GGCYTATCCT TAACACTCCC T) 07 ACACAACCCT GAAAYTGACC ATGAATTTAG GCTTTTTTGA CCAATTTATA AGCCCTACTA TACTAGGATA TACTAGAT CCATTAATT GGCYTAGCCC TTACACTTCC 12 ACACAACCCT GAAGCTGACC ATGAACTTAG GCTTTTTTGA CCAATTTATA AGCCCTACTA TACTAGGAGT CCCATTAATT GGCYTAGCCC TTACGCTTCC

05 GTGACTAATA TTCCCTAAAA CAACTAATCA TTGACTAAAT ATCGACCTCT CAACCAAACA AACTTGATTC TTCGGACTAT TTACAAAACA ACTTATACTC 07 ATGATTACTG TTTCCTAANA CAACTAACCA TTGGTTAACC AACCGCCTAT CAACAACACA AACTTGGCTC TTTGGTATAT TTACTAAACA ACTTATACTT 10 ATA TTGGCTAAAT AACCGCCTAT CAACCACACA AACTTGGTTC TTTGGTATAT TTACTAAGCA GCTTATGCTC

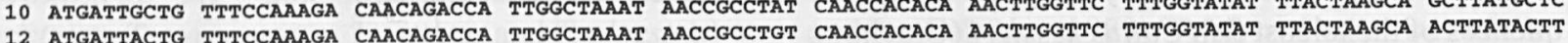

05 CCAATTAATA TT

07 CCAATTGGCG TT

10 CCAATTGGCA TT

12 CCAATTGGCA TT

05 TQPWNWPWI * MNLGFFDQFM SPTVLGIPLI GLSLTLPWLM FPKTTNHWLN IDLSTKQTWF FGLFTKQLML PINI 07 TQPWINPWI* MNLGFFDOFM SPTMLGVPLI GLALTLPWLL FPXTTNHWLT NRLSTTQTWL FGMFTKQLML PIGV 10 TOPWSWPWT* MNLGFFDQFM SPTMLGVPLI GLALTLPWLL FPKTTDHWLN NRLSTTQTWF FGMFTKQLML PIGI 12 TQPWSWPWT* MNLGFFDQFM SPTMLGVPLI GLALTLPWLL FPKTTDHWLN NRLSTTQTWF FGMFTKQLML PIGI

Fig. 2. mtDNA sequence data for $12 \mathrm{~S}$ rRNA (top panel), ATPase (middle panel) and ATPase amino acid sequences (lower panel), with overlap between ATPase genes. DNA sequences are light strands, from $5^{\prime}$ to $3^{\prime}$ end. The taxa studied are : 1) Cynops ensicauda, 2) Neurergus strauchii, 3) Paramesotriton sp., 4) Triturus alpestris, 5) T. boscai, 6) T. cristatus, 7) T. helveticus, 8) $T$. italicus, 9) $T$. marmoratus, 10) T. montandoni, 11) T. vittatus, and 12) T. vulgaris. Asterisks indicate the termination codon for the ATPase 8 gene.

published sequences were added to the data set, lengthening the fragment to 305 positions, with no regions of ambiguous alignment (sensu Titus and Larson, 1995).

One-hundred and sixty-one of the 212 ATPase nucleotide positions were identical across the four species studied and 38 variable positions differed by a single substitution in one taxon (Fig. 2). This left $13(6.1 \%)$ nucleotide positions with potentially, phylogenetically relevant information. The sequence difference between $T$. boscai and in-group taxa ranged between $18.9 \%$ and $19.3 \%$. Within 
the vulgaris species group the distances were considerably lower, ranging from $3.3 \%$ between $T$. montandoni and T. vulgaris to $10.4 \%$ between $T$. montandoni and $T$. helveticus. Taking the short stretch of sequence overlap between both ATPase genes into account, the transition: transversion ratio among in-group taxa was 3.2 and the number of substitutions at the first, second, and third codon position were 5,3 , and 17 , respectively. Following these relationships, the weights assigned to transitions versus transversions were 3 and 21 whereas the weights applied to the first, second and third codon positions were 8,14 , and 2 . At the level of amino acid codon usage, 16 variable characters were found with nine synapomorphic character states for the $T$. helveticus $-T$. montandoni - I. vulgaris clade, four autapomorphic character states for $T$. helveticus and three synapomorphies were found for $T$. montandoni $-T$. vulgaris. The DNA sequences of $T$. vulgaris and T. montandoni differed only by silent substitutions.

Two-hundred and thirty-five of the 301 nucleotide positions on the $12 \mathrm{~S}$ fragment were identical across the 12 taxa studied and 25 variable positions differed by a single substitution in one taxon, leaving $41(13.6 \%)$ phylogenetically informative sites (Fig. 2). Half the number of variable sites were found in one-third of the $12 \mathrm{~S}$ fragment, at the $3^{\prime}$ end. The sequence difference between out-group and in-group taxa ranged between $6.0 \%$ and $14.0 \%$. Within the genus Triturus the distances were considerably lower, ranging from $0.7 \%$ between $T$. montandoni and $T$. vulgaris [which is less than that found within T. alpestris $(3.0 \%)$ and T. vittatus (1.7\%)] to $10.6 \%$ between $T$. cristatus and $T$. alpestris. The average sequence difference between in-group species and an out-group taxon ranged from $7.4 \%$ (Cynops) to $11.2 \%$ (Neurergus). The transition : transversion ratio among in-group taxa was 2.8 and weights determined were 4 and 20.

Given Cynops, Neurergus and Paramesotriton as out-groups, the monophyly of Triturus appears to be strongly supported with a $89 \%$ bootstrap replication score $(P \mathrm{~b})$ (Fig. 3). Two major in-group clusters are formed. The first, with $P \mathrm{~b}=85 \%$, consists of $T$. vittatus as a sister taxon to the $T$. marmoratus species group $(\mathrm{Pb}=98 \%)$. The sec-

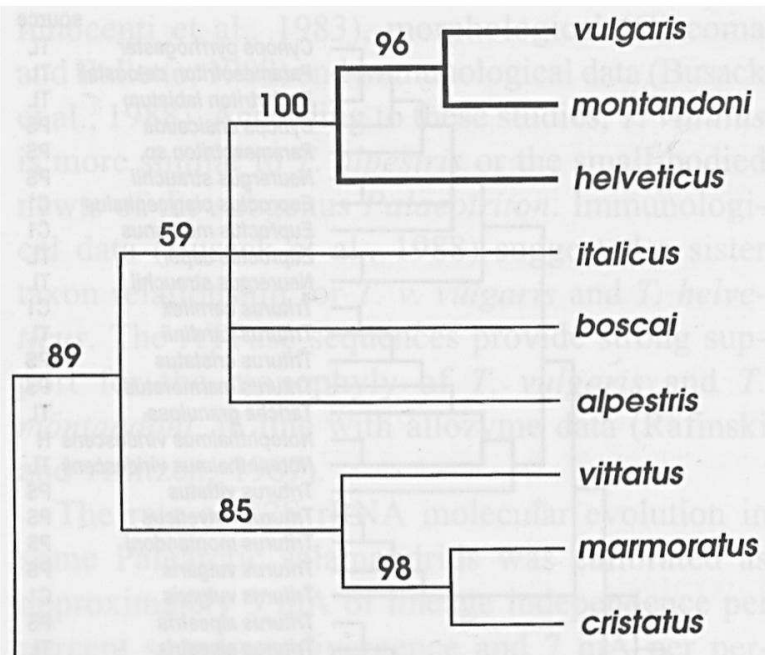

outgroup Cynops, Neurergus, Paramesotriton

Fig. 3. Composite phylogeny for the genus Triturus, constructed from mitochondrial DNA sequence data. Thin and thick lines represent branching order as reconstructed from 12S rRNA and ATPase sequence data, respectively, with bootstrap replication scores indicated along branches.

ond group contains $T$. alpestris and all small-bodied Triturus species, with a low bootstrap support $(P \mathrm{~b}=59 \%)$. Within this group, the monophyly of the T. vulgaris species group is strongly supported $(P \mathrm{~b}=100 \%)$, whereas the relationship of $T$. alpestris, $T$. boscai, and T. italicus among themselves is poorly resolved $(P \mathrm{~b}<55 \%)$. On the basis of $12 \mathrm{~S}$ sequence data no firm conclusion can be drawn regarding the branching order within the vulgaris species group. However, the ATPase sequence data provided strong support for the sister taxon status of $T$. montandoni and $T$. vulgaris ( $\mathrm{Pb}=96$, Fig. 3) and the same bootstrap value was observed when amino-acid sequence data were analyzed instead of the DNA sequence. Under the weighting scheme topologically similar results were obtained, with three reservations: 1) with the ATPase gene fragment, a score of $P \mathrm{~b}=100$ was observed for the vulgaris - montandoni clade, while not with the 12S rRNA gene fragment; 2) generally less phylogenetic resolution and lower bootstrap replication scores were observed on well-established branches; and, 3) an equally parsimonious solution was found in which the genus Triturus is paraphyletic. Sequences derived from the same or congeneric species by different authors (Caccone et al., 1994; 


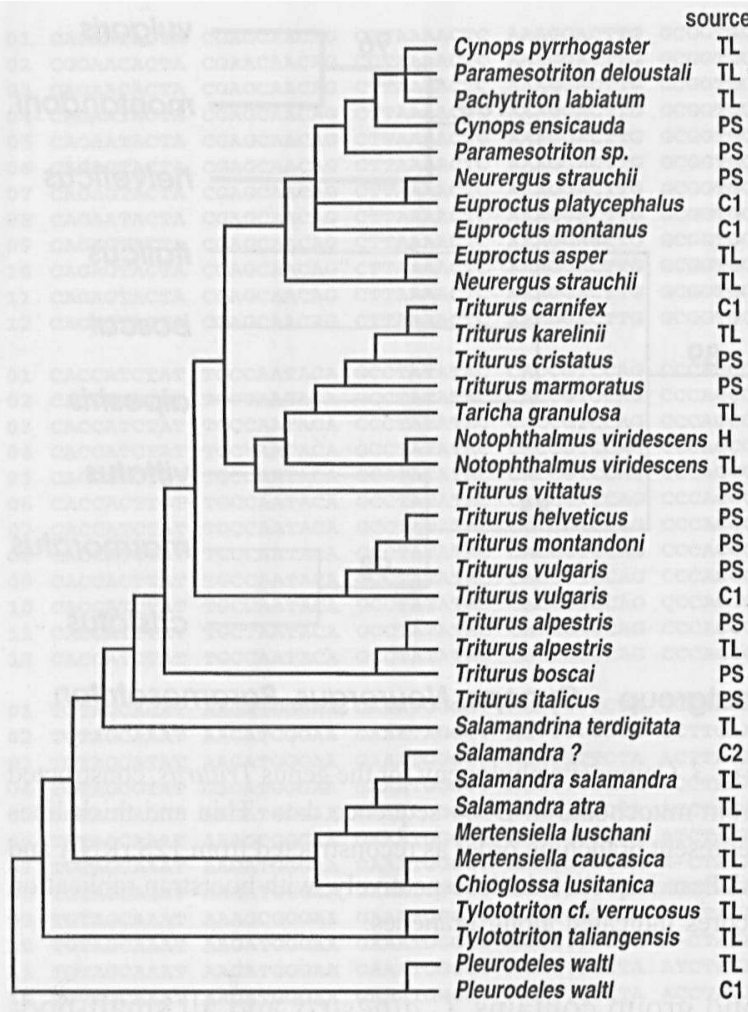

A

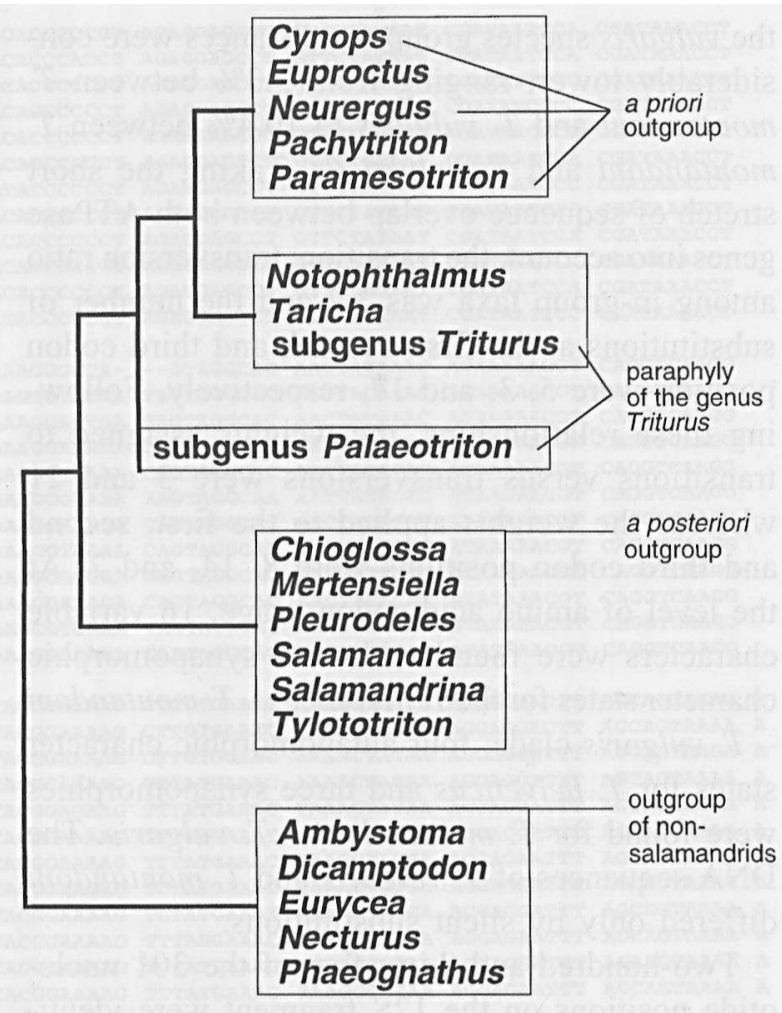

B

Fig. 4. (A) Molecular phylogeny for salamandrid salamanders reconstructed from $12 \mathrm{~S}$ rRNA sequence data as published by $\mathrm{Cl}$, Caccone et al. (1994); C2, Caccone et al. (1994; 523); H, Hay et al. (1995); TL, Titus and Larson (1995) and PS, present study. (B) Schematic phylogeny for salamandrid genera as derived from $12 \mathrm{~S}$ and $16 \mathrm{~S}$ rRNA sequence data (adapted from Titus and Larson, 1995). Note the possible paraphyly of the genus Triturus.

Titus \& Larson, 1995; Hay et al., 1995 and present study) are very similar to one another and consequently the taxa they represent are placed close together in the phylogenetic tree that has maximum parsimony (i.e., Cynops sp., Neurergu $\$$ strauchii, Notophthalmus viridescens, Paramesotriton sp., Pleurodeles waltl, Salamandra salamandra, Triturus alpestris, $T$. cristatus superspecies and $T$. vulgaris).

With a wide range of out-groups included in the analysis, the monophyly of the genus Triturus was not supported. The sister clade to the subgenus Palaeotriton would not be the subgenus Triturus (i.e., $T$. vittatus, $T$. marmoratus, and the $T$. cristatus superspecies), but the subgenus Triturus plus Taricha and Notophthalmus and the sister clade to this group would be composed of Cynops, Euproctus, Neurergus, Pachytriton, and Paramesotriton (Fig. 4A). Templeton's test indicated that the difference between the shortest tree and the tree in which Triturus is monophyletic was significant $(P<0.05)$.

\section{Discussion}

We have tested an existing phylogeny of Triturus against independent molecular data. Two PCRamplified fragments of the mtDNA molecule with relatively low (12S rRNA) and high rates of evolution (ATPase) were employed to test some of the earlier and more recent speciation events of the Triturus radiation, respectively. The chosen fragments showed evolutionary rates that were anticipated and that were appropriate for addressing phylogenetic questions at this taxonomic level. Faster evolving protein-coding ATPase genes can be reliable tracers of evolutionary history among 
close relatives (as within the $T$. vulgaris species group), where silent, third codon position substitutions account for most variation (Zardoya and Meyer, 1996). On the other hand, if the saturation of transitions is suspected, it is imperative to reduce the emphasis on, or even eliminate, this class of substitutions from phylogenetic analysis (Disotell et al., 1992; Knight and Mindell, 1993). Although intuitively appealing, differential weighting may downweight informative transitions in conserved regions and upweight transversions primarily present in more variable regions of the molecule, thereby obscuring phylogenetic relationships (Titus and Larson, 1995; Mindell and Tacker, 1996). Given the observed transition bias, saturation was not prevalent among the compared sequences. The observation that with weighting the bootstrap support for congruent sections of competing phylogenetic solutions dropped and the phylogenetic resolution decreased, supports this view.

Our analysis presents a hypothesis that is concordant with well-supported areas in the established Triturus phylogeny. As to the specific questions we raised, some appear to be solved while others are not. The new data do not help to elucidate the relationship between $T$, boscai and $T$. italicus. Their monophyly was suggested by allozyme data and, ambiguously, by behavioral data (Arntzen and Sparreboom, 1989). The observation of "flamenco" behavior in the sexual repertoire of $T$. helveticus (M. Faria, pers. comm.) and T. marmoratus pygmaeus (T. Halliday, pers. comm., M. Sparreboom, pers. comm.) further erodes the support for T. boscai $-T$. italicus monophyly. Similarly, the current study does not convincingly clarify the position of $T$. alpestris. The phylogenetic position of this species could also not be satisfactorily resolved from behavioral data (Arntzen and Sparreboom, 1989). Bolkay (1928) placed it in a third subgenus with intermediate characteristics, in between Triturus and Palaeotriton.

The 12S rRNA sequence data support the placement of $T_{*}$ vittatus as the sister taxon to the $T$. marmoratus species group in the subgenus Triturus, as was inferred from its breeding behavior (Arntzen and Sparreboom, 1989). This challenges the inferences from the phenetic analyses of osteological (Rafinski and Pecio, 1989), karyological (Bucci-
Innocentí et al., 1983), morphological (Gíacoma and Balletto, 1988) and immunological data (Busack et al., 1988). According to these studies, $T$, vittatus is more similar to $T$, alpestris or the small-bodied newts of the subgenus Palaeotriton. Immunological data (Busack et al., 1988) suggested a sister taxon relationship for $T, v$. vulgaris and $T$. helveticus. The ATPase sequences provide strong support for the monophyly of $T$. vulgaris and $T$. montandoni, in line with allozyme data (Rafinski and Arntzen, 1987).

The rate of 12S rRNA molecular evolution in some Palearctic salamandrids was calibrated as approximately $3 \mathrm{~mA}$ of lineage independence per percent sequence divergence and $7 \mathrm{~mA}$ per percent sequence divergence when only transversions are considered (Caccone et al., 1994). Applying these rates provides an estimate of 12-19 $\mathrm{mA}$ for the age of the $T$. vittatus lineage (versus the $T$. marmoratus species group) in the subgenus Triturus and an estimate of 14-19 $\mathrm{mA}$ for the $T$. boscai lineage (versus the $T$. vulgaris species group) within the subgenus Palaeotriton. This is in line with estimates from fossil, biochemical, molecular and biogeographical data that converge to $14-15 \mathrm{~mA}$ for the T. vittatus lineage and to 13-15 $\mathrm{mA}$ for the T. boscai lineage (reviewed in Oosterbroek and Arntzen, 1992).

The monophyly of the genus Triturus, although widely accepted, is defined on the basis of somewhat vague character state descriptions ('a suite of behavioral character states' and 'a high level of sexual dimorphism* - Halliday, 1977) that are not explicit synapomorphies. Moreover, a feature such as the potential for interbreeding (Wolterstorff and Herre, 1935) is explicitly plesiomorphic. The monophyly of the genus Triturus has recently been put into question in a molecular phylogenetic study of the family Salamandridae (Titus and Larson, 1995) and in a molecular biogeographical study of Euproctus (Caccone et al,, 1994). In both of them only two Triturus species were involved. With just the representatives of the genera Cynops, Neurergus and Paramesotriton as out-groups, a denser in-group taxon sampling does not challenge the hypothesis of Triturus monophyly. However, $12 \mathrm{~S}$ and $16 \mathrm{~S}$ mtDNA sequence data indicate that the genera Cynops, Neurergus and Paramesotriton 
themselves may be in-group taxa relative to Triturus (Fig. 4B). In the light of these results, their a priori choice as out-groups to the genus Triturus may have been unfortunate.

\section{Acknowledgements}

We acknowledge the laboratory facilities provided by Prof. $T$ * Burke, the assistance of Dr. D. Thomaz, and the constructive comments of Drs. A. Zuiderwijk and an anonymous reviewer.

\section{Literature}

Arnason U, Xu X, Gullberg A. 1996. Comparison between the complete mitochondrial DNA sequences of Homo and the common chimpanzee based on nonchimeric sequences. $J$. Mol. Evol. 42: 145-152.

Arntzen JW, Sparreboom M. 1989. A phylogeny of the Old World newts, genus Triturus: biochemical and behavioural data. J. Zool (Lond.) 219: 645-664.

Bolkay SJ. 1928. Die Schädel der Salamandrinen, mit besonderer Rückssicht auf ihre systematissche Bedeutung. Zeitschr. Anat. Entwickelungsgesch. 86: 259-319.

Bucei-Innocenti S, Ragghianti M, Mancino G. 1983. Investigations of karyology and hybrids in Triturus boscai and $T$. vittatus, with reinterpretation of the species groups within Triturus (Caudata: Salamandridae), Copeia 1983: 662-672.

Busack SD, Jericho BG, Maxson LR, Uzzell T. 1988. Evolutionary relationships of salamanders in the genus Triturus: The view from immunology. Herpetologica 44: 307-316.

Caccone A, Milinkovitch MC, Sbordoni V, Powell JR. 1994. Molecular biogeography: using the Corsica-Sardinia microplate disjunction to calibrate mitochondrial rDNA evolutionary rates in mountain newts (Euproctus). Erratum. $J$. Evol. Biol. 7: 227-245, 523-524.

Caceone A, Milinkovitch MC, Sbordoni V, Powell JR. 1997. Mitochondrial DNA rates and biogeography in European newts (genus Euproctus), Syst. Biol, 46: 126-144.

Disotell T R, Honeycutt RL, Ruvolo M. 1992. Mitochondrial DNA phylogeny of the Old-World monkey tribe Papionini. Mol. Biol. Evol. 9: 1-13.

Erlich HA (ed.). 1989. PCR Technology. Principles and Applications for DNA amplification. New York: Stockton Press.

Felsenstein J. 1985. Confidence limits on phylogenies: An approach using the bootstrap. Evolution 39: 783-791.

Giacoma C, Balletto E. 1988. Phylogeny of the salamandrid genus Triturus. Boll. Zool. 55: 337-360.

Grachev MA, Slobodyanyuk SJ, Kholodilov NG, Fyodorov SP, Belikov SI, Sherbakov DY, Sideleva VG, Zubin AA, Kharchenko VV. 1992. Comparative study of two proteincoding regions of mitochondrial DNA from three endemic sculpins (Cottoidei) of Lake Baikal. J. Mol. Evol. 34: 8590.
Halliday TR.1977. The courtship of European newts: an evolutionary perspective. In: Taylor DH, Gutman SE, eds, The reproductive biology of amphibians. New York: Plenum Press, 185-232.

Halliday T, Arano B. 1991. Resolving the phylogeny of the European newts. Trends Ecol. Evol. 6: 107-117.

Hay JM, Ruvinsky I, Hedges SB, Maxson LR. 1995. Phylogenetic relationships of amphibian families inferred from DNA sequences of mitochondrial $12 \mathrm{~S}$ and $16 \mathrm{~S}$ ribosomal RNA genes. Mol. Biol. Evol. 12: 928-937.

Hickson RE, Simon C, Cooper A, Spicer GS, Sullivan J, Penny D. 1996. Conserved sequence motifs, alignment, and secondary structure for the third domain of animal 12S rRNA. Mol. Biol. Evol. 13: 150-169.

Higgins DG, Sharp PM. 1988. CLUSTAL; a package for performing multiple sequence alignment on a microcomputer. Gene 73: 237-244.

Hillis DM, Bull JJ. 1993. An empirical test of bootstrapping as a method for assessing confidence in phylogenetic analysis. Syst. Biol. 42: 182-192.

Knight A, Mindell DP. 1993. Substitution bias, weighting of DNA sequence evolution and the phylogenetic position of Fea's viper. Syst. Biol. 42: 18-31.

Kocher TD, Thomas WK, Meyer A, Edwards SW, Päăbo S, Villablanca FX, Wilson AC. 1989. Dynamics of mitochondrial DNA evolution in animals: Amplification and sequencing with conserved primers. Proc. Nath. Acad. Sci. U.S.A. 86: 6196-6200.

Kumar S. 1996. Patterns of nucleotide substitution in mitochondrial protein coding genes of vertebrates. Genetics 143: 537-548.

Macgregor HC, Sessions SK, Arntzen JW. 1990. An integrative analysis of phylogenetic relationships among newts of the genus Triturus (family Salamandridae), using comparative biochemistry, cytogenetics and reproductive interactions. J. Evol. Biol. 3: 329-373.

Mindell DP, Honeycutt RL. 1990. Ribosomal RNA in vertebrates: Evolution and phylogenetic implications. Annu. Rev. Ecol. Syst. 21: 541-566.

Mindell DP, Tacker CE. 1996. Rates of molecular evolution: phylogenetic issues and applications, Annu. Rev. Ecol. Syst. 27: 279-303.

Oosterbroek P,Arntzen JW. 1992. Area-cladograms of circumMediterranean taxa in relation to Mediterranean palaeogeography. J. Biogeogr. 19: 3-20.

Rafinski J, Pecio A. 1989. Craniometric studies on the species of the genus Triturus, Rafinesque, 1815 (Amphibia, Salamandridae). Folia Biol. 37: 131-150.

Rafinski J,Arntzen JW. 1987. Biochemical systematics of the Old World newts, genus Triturus: allozyme data. Herpetologica 43: 446-457.

Sambrook J, Fritsch EF, Maniatis T. 1989. Molecular cloning: A laboratory manual. New York: Cold Spring Harbor Laboratory Press.

Sanger F, Nickeln S, Coulson AR. 1977. DNA sequencing with chain-terminating inhibitors. Proc. Natl. Acad. Sci. U.S.A. 74: 5463-5467. 
Swofford DL. 1991. PAUP - phylogenetic analysis using parsimony, version 3.1. Champaign: Illinoís Natural Hístory Survey.

Templeton AR. 1983. Phylogenetic inference from restriction endonuclease cleavage site maps. with particular reference to the evolution of humans and apes. ${ }^{x}$ Evolution 37: 221244.

Titus TA, Larson A. 1995. A molecular phylogenetic perspective on the evolutionary radiation of the Salamander family Salamandridae. Syst. Biol. 44: 125-151.

Wallis GP, Arntzen JW. 1989. Mitochondrial DNA variation in the crested newt superspecies: Limited cytoplasmic gene flow among species. Evolution 43: 88-104.
Wolterstorff W, Herre W. 1935. Die Gattungen der Wassermolche der Familie Salamandridae. Archiv Naturgesch. 4: 217-229.

Zardoya R, Meyer A. 1996. Phylogenetic performance of mitochondrial protein-coding genes in resolving relationships among vertebrates. Mol. Biol. Evol. 13; 933-942.

Zhang D-X, Hewitt GM. 1996. Nuclear integrations: challenges for mitochondrial DNA markers. Trends Ecol. Evol. 11: 247251.

Received: 4 March 1998 